\title{
The Relationship between Nuclear Maturation and Cortical Granule Distribution in Cultured Porcine Oocytes
}

\author{
Hiroko Takano ${ }^{1}$, Chika Kanda ${ }^{2}$ and Sueo Niimura ${ }^{2 *}$ \\ ${ }^{1}$ Graduate School of Science and Technology, and \\ ${ }^{2}$ Faculty of Agriculture, Niigata University, Niigata 950-2181, Japan
}

\begin{abstract}
The state of nuclear maturation and the state of cortical granule (CG) distribution were observed in porcine oocytes cultured for various periods and also in those treated with olomoucine, an inhibitor of cyclindependent kinase, and the relationship between nuclear maturation and changes in CG distribution was investigated. CGs were distributed over the cortical cytoplasm (type I) in $98 \%$ of porcine oocytes soon after collection and in $90 \%$ of those cultured for 8 hrs after collection, and nuclei of these oocytes were all in the germinal vesicle (GV) stage. When cultured for 22 hrs, type l oocytes markedly decreased, while oocytes having CGs in the cortical cytoplasm and also immediately beneath the plasma membrane (type II) increased to $22 \%$, and oocytes having CGs immediately beneath the plasma membrane (type III) appeared at $72 \%$. Nuclei of type II oocytes were in the GV to M I stages, while most nuclei of type III oocytes were in the M I stage. When cultured for 32 and 44 hrs, type III oocyte populations were observed as 70 and $87 \%$ of the whole, respectively, and nuclei of these type III oocytes were all in the $M I$ to $M I I$ stages. In the oocytes cultured for 22 hrs with olomoucine, nuclei were all in the GV stage, and $83 \%$ of the treated oocytes showed the type I distribution of CGs. On the other hand, $91 \%$ of control oocytes showed the type II and type III distribution of CGs, and type I oocyte populations were observed only as $10 \%$ of the whole. From these results, it was inferred in porcine oocytes that the start and the completion of CG movement into the cytoplasm immediately beneath the plasma membrane are closely related to the time at which GV breaks down and nuclei reach the M I stage, respectively.
\end{abstract}

Key words: Porcine oocyte, Nuclear maturation,

Received: December 7, 2001

Accepted: February 7, 2002

*To whom correspondence should be addressed.

e-mail: niimura@agr.niigata-u.ac.jp
Cortical granule distribution, Lectin histochemistry, Olomoucine

Changes in cortical granule (CG) distribution with maturation have been studied in porcine oocytes under electron microscopy [1, 2] and also histochemically with lectin [2-4]. CGs are distributed over the cortical cytoplasm in the germinal vesicle (GV)-stage oocyte collected from antral follicles, and the CGs move to the cytoplasm immediately beneath the plasma membrane as maturation progresses [1-4]. In oocytes collected from antral follicles $20 \mathrm{hrs}$ [1] or 24 to $36 \mathrm{hrs}$ [2] after hCG injection and those cultured for $24 \mathrm{hrs}$ [2] or $26 \mathrm{hrs}$ $[3,4]$, most CGs are observed immediately beneath the plasma membrane.

When porcine oocytes cultured for $26 \mathrm{hrs}$, in which CGs are distributed immediately beneath the plasma membrane, were inseminated, sperm penetration was observed in $98 \%$ of the oocytes, and $81 \%$ of those oocytes showed polyspermy, but only $26 \%$ of the oocytes could completely release CGs after insemination [4]. Since a high frequency of polyspermy and poor cortical reaction were observed after insemination, even in oocytes in which CGs had completely moved to the cytoplasm immediately beneath the plasma membrane [4], oocytes do not always sufficiently mature, even if CGs are distributed immediately beneath the plasma membrane. Therefore, it is important to clarify the relationship between nuclear maturation and changes in $C G$ distribution in porcine oocytes.

In the present study, the state of nuclear maturation and the state of CG distribution were observed in porcine oocytes cultured for various periods in order to investigate the relationship between nuclear maturation and changes in CG distribution in oocytes. The state of 
CG distribution was also examined in oocytes treated with olomoucine [5], an inhibitor of cyclin-dependent kinase such as maturation-promoting factor (MPF).

\section{Materials and Methods}

\section{Collection and culture of oocytes}

Ovaries were obtained from prepubertal gilts at a local slaughterhouse and transported to the laboratory in $0.9 \% \mathrm{NaCl}$ solution maintained at $37^{\circ} \mathrm{C}$. The ovaries were washed in $0.9 \% \mathrm{NaCl}$ solution containing 200 i.u./ $\mathrm{ml}$ potassium penicillin G. Immature oocytes covered with cumulus cells were aspirated from medium-sized follicles (3-6 $\mathrm{mm}$ in diameter) with a 21-gauge needle fixed to a 10-ml disposable syringe. Collected cumulusoocyte complexes (COCs) were washed in phosphate buffered saline (PBS, pH 7.4) [6] and then in TCM-199 (Gibco BRL, NY, USA) [7]. Forty to fifty COCs were transferred into each well of a Nunc 4-well multidish (Nunc, Roskilde, Denmark) containing $400 \mu$ of TCM199 , which had previously been covered with mineral oil (Squibb and Sons, NJ, USA) and equilibrated in ${\mathrm{a} \mathrm{CO}_{2}}$ incubator. These COCs were cultured at $39^{\circ} \mathrm{C}$ in an atmosphere of $5 \% \mathrm{CO}_{2}$ in air.

\section{In vitro fertilization of oocytes}

Ejaculated boar semen was treated by the method of Wang et al. [8], in order to induce capacitation of spermatozoa. The semen was washed three times in Brackett and Oliphant (BO) [9] medium containing $5 \mathrm{mM}$ caffeine (Sigma Chemical Co., MO, USA) and 0.3\% BSA (Sigma Chemical Co.). Spermatozoa were resuspended in the $\mathrm{BO}$ medium to give a concentration of $5 \times 10^{5}$ live spermatozoa $/ \mathrm{ml}$, and $400 \mu \mathrm{l}$ of sperm suspension were covered with mineral oil in each well of a Nunc 4-well multidish.

At 44 hrs after maturation culture, COCs were washed twice in the BO medium. Forty to fifty COCs were introduced into the sperm suspension and cultured at $39^{\circ} \mathrm{C}$ in a $\mathrm{CO}_{2}$ incubator $\left(5 \% \mathrm{CO}_{2}\right.$ in air).

\section{Observation of CG distribution}

At $0,8,22,32$ and 44 hrs after maturation culture, cumulus cells were dispersed from the oocytes by pipetting in PBS containing $0.1 \%$ hyaluronidase (Sigma Chemical Co.). The denuded oocytes were stained at various times after maturation culture and at $8 \mathrm{hrs}$ after insemination with PNA conjugated with FITC (E-Y Lab., CA, USA) according to the method of Ducibella et al. [10]. The denuded oocytes were immersed in PBS containing $0.2 \%$ pronase (Sigma Chemical Co.) to dissolve the zonae pellucidae. The naked oocytes were washed in PBS and fixed in PBS containing $3.7 \%$ paraformaldehyde for $30 \mathrm{~min}$ at room temperature. They were then washed 3 times in a blocking solution composed of $3 \mathrm{mg}$ BSA, $7.51 \mathrm{mg}$ glycine (Wako Pure Chemical, Osaka, Japan) and $1 \mathrm{ml}$ PBS, immersed in PBS containing $0.1 \%$ Triton $\mathrm{X}-100$ (Nacalai Tesque, Kyoto, Japan) for $5 \mathrm{~min}$ at $20^{\circ} \mathrm{C}$, and again placed in the blocking solution. These oocytes were finally immersed in a staining solution composed of $100 \mu \mathrm{g}$ PNA, $0.1 \mu \mathrm{l}$ Triton X-100, $3 \mathrm{mg} \mathrm{BSA}$ and $1 \mathrm{ml}$ PBS for $30 \mathrm{~min}$ at $20^{\circ} \mathrm{C}$. The stained oocytes were thoroughly washed in PBS containing $0.01 \%$ Triton $\mathrm{X}-100$ and $0.3 \%$ BSA, and were placed in the center of four vaseline spots on a slide. A coverslip was then carefully placed on the vaseline spots and pressed gently to anchor the oocyte between the coverslip and the slide. Observation was carried out under an epifluorescence microscope (Nikon, Tokyo, Japan). Degenerated oocytes were eliminated from the observation.

\section{Observation of nuclear maturation}

In order to investigate nuclei of oocytes cultured for various periods, oocytes in which CGs had been observed were fixed in $25 \%(\mathrm{v} / \mathrm{v})$ acetic acid in ethanol for $48 \mathrm{hrs}$ at $4^{\circ} \mathrm{C}$. The fixed oocytes were stained with $1.0 \%$ aceto-orcein and examined for evidence of nuclear maturation under a light microscope.

Observation of CG distribution and nuclear maturation in olomoucine-treated oocytes

To observe the state of CG distribution in oocytes in which resumption of meiotic division was inhibited, COCs collected from antral follicles were cultured for 22 hrs at $39^{\circ} \mathrm{C}$ in TCM-199 containing olomoucine (Sigma Chemical Co.) at $400 \mu \mathrm{M}$. Olomoucine was previously dissolved in DMSO and then diluted with the culture medium up to $400 \mu \mathrm{M}$. The concentration of DMSO in the culture medium was adjusted to $0.37 \%$. Oocytes cultured in the medium containing DMSO at $0.37 \%$ for 22 hrs were used as controls. After culture, PNA staining was performed, as mentioned previously and then the state of CG distribution was observed. To ensure the inhibition of resumption of meiotic division by olomoucine treatment, oocytes in which CGs had been observed were fixed in $25 \%$ (v/v) acetic acid in ethanol, stained with $1.0 \%$ aceto-orcein and then examined under a light microscope. To determine the viability of the olomoucine-treated oocytes, progression of nuclear maturation was also observed in those further cultured for $44 \mathrm{hrs}$ in the medium without olomoucine. 

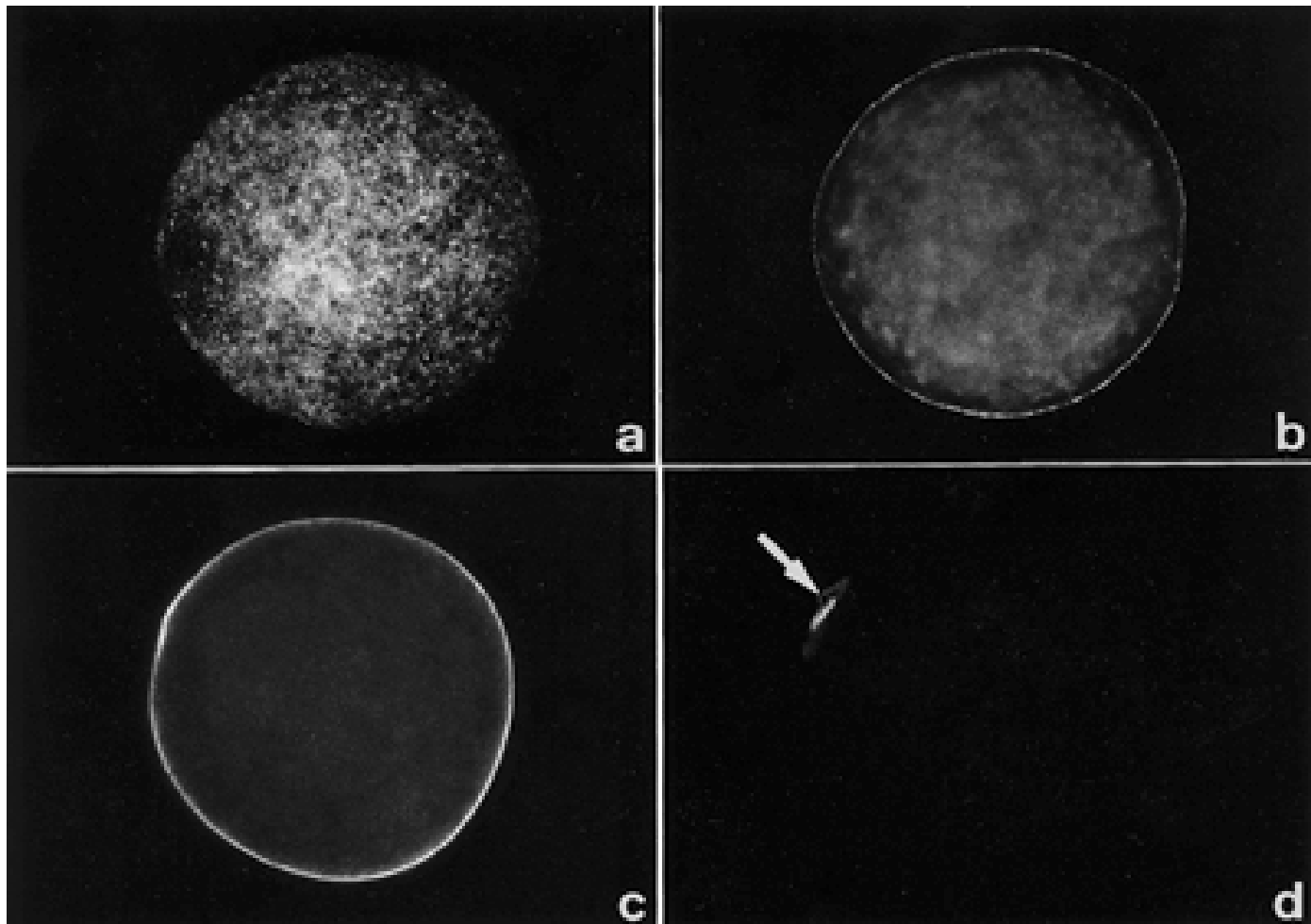

Fig. 1. Whole mount preparations of porcine oocytes with a magnification of $\times 400$ under an epifluorescence microscope. All oocytes were photographed after staining with FITC-conjugated PNA, and the images were taken at the superficial (a) and the equatorial (b, c, d) portions of cytoplasm.

a. A type I oocyte at the germinal vesicle stage just after collection. Cortical granules are distributed over the cortical cytoplasm. b. A type II oocyte $8 \mathrm{hrs}$ after maturation culture. Cortical granules are distributed in the cortical cytoplasm and also immediately beneath the plasma membrane. c. A type III oocyte $32 \mathrm{hrs}$ after maturation culture. Cortical granules are densely distributed immediately beneath the plasma membrane. d. A fertilized oocyte $8 \mathrm{hrs}$ after insemination. No cortical granules are seen in the cytoplasm. First polar body (arrow) is seen.

\section{Statistical analysis}

The rates concerning nuclear maturation and the number of oocytes with different distribution patterns of CGs were statistically analyzed by Chi-square test.

\section{Results}

When porcine oocytes cultured for various periods were stained with PNA, fluorescent granules appeared in their cytoplasm (Fig. $1 \mathrm{a}-\mathrm{c}$ ). Since these granules completely disappeared in $83 \%(50 / 60)$ of inseminated oocytes (Fig. 1d), the PNA-reactive granules were determined to be CGs. Distribution patterns of CGs differed among oocytes cultured for different periods and could be classified into 3 types. In type I, CGs were distributed over the cortical cytoplasm (Fig. 1a). In type II, CGs were distributed in the cortical cytoplasm and also immediately beneath the plasma membrane (Fig. 1b). CGs in oocytes of type III were all densely distributed just beneath the plasma membrane (Fig. 1c). Immediately after collection and after culture for $8 \mathrm{hrs}$, 98 and $90 \%$ of oocytes respectively showed type I CG distribution, and the remaining oocytes were all classified as type II (Table 1). Nuclei of these oocytes showing type I or II distribution of CGs were all in the GV stage. At 22 hrs after culture, type I oocytes decreased to $6 \%(3 / 54)$, while type II oocytes increased to $22 \%(12 / 54)$ and type III oocytes first appeared at $72 \%(39 / 54)$. Nuclei of the type II oocytes were in the $\mathrm{GV}$, diakinesis and M I stages. Most nuclei of the type III oocytes were in the M I stage. Of oocytes cultured for 32 and $44 \mathrm{hrs}, 70(40 / 57)$ and $87 \%$ (41/47) were respectively found to be type III, and their nuclei were all in the M I to M II stages. 
Table 1. The changes in CG distribution and nuclei of cultured porcine oocytes

\begin{tabular}{|c|c|c|c|c|c|c|c|c|c|c|c|c|c|c|}
\hline \multirow{3}{*}{$\begin{array}{c}\text { Hours } \\
\text { of } \\
\text { culture }\end{array}$} & \multirow{3}{*}{$\begin{array}{c}\text { No. of } \\
\text { oocytes } \\
\text { examined }\end{array}$} & \multicolumn{13}{|c|}{ No. and (\%) of oocytes with different types* of CG distribution } \\
\hline & & \multicolumn{3}{|c|}{ I } & \multicolumn{5}{|c|}{ II } & \multicolumn{5}{|c|}{ III } \\
\hline & & GV & Dia & Total & GV & Dia & MI & MII & Total & Dia & MI & TI & MII & Total \\
\hline 0 & 61 & $60(98)$ & $0(0)$ & $60(98)$ & $1(2)$ & $0(0)$ & $0(0)$ & $0(0)$ & $1(2)$ & $0(0)$ & $0(0)$ & $0(0)$ & $0(0)$ & $0(0)$ \\
\hline 8 & 62 & $56(90)$ & $0(0)$ & $56(90)$ & $6(10)$ & $0(0)$ & $0(0)$ & $0(0)$ & $6(10)$ & $0(0)$ & $0(0)$ & $0(0)$ & $0(0)$ & $0(0)$ \\
\hline 22 & 54 & $1(2)$ & 2(4) & $3(6)$ & $4(7)$ & $1(2)$ & $7(13)$ & $0(0)$ & $12(22)$ & $1(2)$ & $33(61)$ & $0(0)$ & $5(9)$ & $39(72)$ \\
\hline 32 & 57 & $3(5)$ & $0(0)$ & $3(5)$ & $2(4)$ & $0(0)$ & $4(7)$ & $8(14)$ & $14(25)$ & $0(0)$ & $12(21)$ & $3(5)$ & $25(44)$ & $40(70)$ \\
\hline 44 & 47 & $0(0)$ & $0(0)$ & $0(0)$ & $0(0)$ & $0(0)$ & $0(0)$ & $6(13)$ & $6(13)$ & $0(0)$ & $9(19)$ & $0(0)$ & $32(68)$ & $41(87)$ \\
\hline
\end{tabular}

*Type I: CGs were distributed over the cortical cytoplasm. Type II: CGs were distributed over the cortical cytoplasm and also immediately beneath the plasma membrane. Type III: CGs were distributed immediately beneath the plasma membrane. GV: Germinal vesicle, Dia: diakinesis, MI: metaphase I, TI: telophase I, MII: metaphase II.

Table 2. The changes in CG distribution and nuclei of porcine oocytes cultured with olomoucine

\begin{tabular}{|c|c|c|c|c|c|c|c|c|c|c|c|c|c|c|}
\hline \multirow{3}{*}{$\begin{array}{l}\text { Concentration } \\
\text { of olomoucine } \\
(\mu \mathrm{M})\end{array}$} & \multirow{3}{*}{$\begin{array}{l}\text { No. of } \\
\text { oocytes } \\
\text { examined }\end{array}$} & \multicolumn{13}{|c|}{ No. and (\%) of oocytes with different types* of CG distribution } \\
\hline & & \multicolumn{4}{|c|}{ I } & \multicolumn{5}{|c|}{ II } & \multicolumn{4}{|c|}{ III } \\
\hline & & GV & Dia & MI & Total & GV & Dia & MI & MII & Total & Dia & MI & MII & Total \\
\hline 0 & 42 & $2(5$ & 1( & $1(2)$ & 4( & $1(2)$ & $1(2)$ & $7(17)$ & $1(2)$ & 10( & $1(2)$ & $22(52)$ & $5(12)$ & $28(67)$ \\
\hline 400 & 36 & $30(83)$ & $0(0)$ & $0(0)$ & $30(83)$ & $6(17)$ & $0(0)$ & $0(0)$ & $0(0)$ & $6(17)$ & $0(0)$ & $0(0)$ & $0(0)$ & $0(0)$ \\
\hline
\end{tabular}

The oocytes were observed after 22 hrs of culture. *Type I: CGs were distributed over the cortical cytoplasm. Type II: CGs were distributed over the cortical cytoplasm and also immediately beneath the plasma membrane. Type III: CGs were distributed immediately beneath the plasma membrane. GV: Germinal vesicle, Dia: diakinesis, MI: metaphase I, MII: metaphase II.

The changes in CG distribution and nuclei of porcine oocytes treated with olomoucine are shown in Table 2. Of oocytes cultured for $22 \mathrm{hrs}$ in the medium containing olomoucine, only $17 \%(6 / 36)$ showed the type II distribution of CGs, and the remaining $83 \%$ were classified as type I. Nuclei of the olomoucine-treated oocytes were all in the GV stage. The percentage of oocytes showing types II and III distribution of CGs was significantly higher in control oocytes $(91 \%, 38 / 42)$ than in the olomoucine-treated oocytes $(17 \%, 6 / 36)$. Nuclei of the control oocytes were in the GV to M II stages, mostly in the M I stage $(71 \%, 30 / 42)$.

When olomoucine-treated oocytes were further cultured in olomoucine-free medium for $44 \mathrm{hrs}$, most nuclei progressed to the $M I I$ stage $(81 \%, 30 / 37)$, suggesting that the ability to mature among olomoucinetreated oocytes was sustained.

\section{Discussion}

In the present study, cultured porcine oocytes were first classified into 3 types according to the state of $C G$ distribution: type I in which CGs were distributed over the cortical cytoplasm, type III in which CGs were distributed immediately beneath the plasma membrane, and type II in which CGs were distributed over both cortical cytoplasm and immediately beneath the plasma membrane. Cran et al. [1] reported that $80 \%$ of CGs were distributed over the cortical cytoplasm in oocytes collected from antral follicles $20 \mathrm{hrs}$ after hCG injection, while $20 \%$ were distributed immediately beneath the plasma membrane. The observation of Cran et al. [1] convinced us that in vitro maturing oocytes, classified as type II in the present study, were in the transient period from type I to type III.

The results of the present investigation revealed that nuclei were all in the GV stage in oocytes 0 and $8 \mathrm{hrs}$ after culture, and most oocytes showed type I distribution of CGs. Type I oocytes with GVs markedly decreased as the time of culture was prolonged, and most of the oocytes showed the type III distribution of CGs after culturing for 22 to $44 \mathrm{hrs}$. In $99 \%$ (119/120) of the type III oocytes, their nuclei were in the M I to M II stages. Since 22-hr culture corresponds to the time of completion of GV breakdown (GVBD) in porcine oocytes [11], GVBD was thought to be closely related to the start of the movement of CGs in the cytoplasm. From the results of the present study, it was inferred that the distribution of CGs altered almost in parallel with nuclear maturation, and CGs moved to the 
cytoplasm immediately beneath the plasma membrane in the M I stage. Since it has been observed that nuclei of oocytes having CGs immediately beneath the plasma membrane reach the $\mathrm{MI}$ I stage in 75 to $89 \%$ of oocytes cultured for $26 \mathrm{hrs}$, and of them $90 \%$ reach the M II stage when cultured for $46 \mathrm{hrs}[3,4]$, the state of nuclear maturation in oocytes showing the type III distribution of CGs observed in the present study agrees with those reported previously.

We also attempted to examine the CG distribution using olomoucine-treated oocytes in order to determine the relationship between nuclear maturation and changes in CG distribution in the cytoplasm. The resumption of nuclear maturation was completely inhibited in the olomoucine-treated oocytes, and the change in distribution of CGs was also inhibited. Therefore, resumption of nuclear maturation in porcine oocytes was determined to be associated with the start of movement of CGs in the cytoplasm. Since microfilaments are involved in the dynamics of CGs in porcine oocytes [12] and it was determined in the present study that GVBD and movement of CGs to the cytoplasm immediately beneath the plasma membrane were significantly inhibited in olomoucine-treated oocytes, MPF is thought to be related to not only GVBD in oocytes but also the dynamics of microfilaments in their cytoplasm. However, the results of the present study do not provide the information needed to identify the interaction between MPF and the dynamics of microfilaments. This issue should be further studied.

In the present study, the type II distribution of CGs could be observed even in oocytes with nuclei in the GV or M II stage. Thus it was thought that nuclear maturation does not always progress in correspondence with changes in CG distribution. In oocytes cultured for $44 \mathrm{hrs}$ in the present study, it was determined that some oocytes showed the type II distribution of CGs, even in the M II stage, while some nuclei did not reach the M II stage in oocytes showing the type III distribution of CGs. Thus it was inferred that the presence of such oocytes is a cause of polyspermy at a high frequency after insemination.

\section{References}

1) Cran, D.G. and Cheng, W.T.K. (1985): Changes in cortical granules during porcine oocyte maturation. Gamete Res.,
11, 311-319.

2) Yoshida, M., Cran, D.G. and Percel, V.G. (1993): Confocal and fluorescence microscopic study using lectins of the distribution of cortical granule during the maturation and fertilization of pig oocytes. Mol. Reprod. Dev., 36, 462468.

3) Wang, W.H., Sun, Q.Y., Hosoe, M., Shioya, Y. and Day, B.N. (1997): Quantified analysis of cortical granule distribution and exocytosis of porcine oocytes during meiotic maturation and activation. Biol. Reprod., 56, 13761382.

4) Wang, W.H., Hosoe, M. and Shioya, Y. (1997): Induction of cortical granule exocytosis of pig oocytes by spermatozoa during meiotic maturation. J. Reprod. Fert., $109,247-255$.

5) Abraham, R.T., Acquarone, M., Andersen, A., Asesi, A., Belle, R., Berger, F., Bergounioux, C., Burnn, G., BuquetFagot, C., Fagot, D., Glab, N., Goudeau, H., Goudeau, M., Guerrier, P., Houghton, P., Hendriks, H., Kloareg, B., Lippai, M., Marie, D., Maro, B., Meijer, L., MulnerLorillon, O., Poulet, S.A., Schierenberg, E., Schutte, B., Vaulot, D. and Verlhac, M.H. (1995): Cellular effect of olomoucine an inhibitor of cyclin-dependent kinases. Biol. Cell., 83, 105-120.

6) Dulbecco, R. and Vogt, M. (1954): Plaque formation and isolation of pure lines with poliomyelitis viruses. J. Exp. Med., 99, 167-174.

7) Yoshida, M., Ishizaki, Y. and Kawagishi, H. (1990): Blastocyst formation by pig embryos resulting from invitro fertilization of oocytes matured in vitro. J. Reprod. Fert., 88, 1-8.

8) Wang, W.H., Abeydeera, L.R., Fraser, L.R. and Niwa, K. (1995): Functional analysis using chlortetracycline fluorescence and in vitro fertilization of frozen-thawed ejaculated boar spermatozoa incubated in a protein-free chemically defined medium. J. Reprod. Fert., 104, 305 313.

9) Brackett, B.G. and Oliphant, G. (1975): Capacitation of rabbit spermatozoa in vitro. Biol. Reprod., 12, 260-274.

10) Ducibella, T., Anderson, E., Albertini, D.F., Aalberg, J. and Rangarajan, S. (1988): Quantitative studies of changes in cortical granule number and distribution in the mouse oocyte during meiotic maturation. Dev. Biol., 130, 184197.

11) Motlik, J. and Fulka, J. (1976): Breakdown of the germinal vesicle in pig oocytes in vivo and in vitro. J. Exp. Zool., 198, 155-162.

12) Sun, Q.Y., Lai, L., Park, K.W., Kuhholzer, B., Prather, R.S. and Schatten, H. (2001): Dynamic events are differently mediated by microfilaments, microtubules, and mitogenactivated protein kinase during porcine oocyte maturation and fertilization in vitro. Biol. Reprod., 64, 879-889. 